\title{
Deformation and fracture of crystalline tungsten and fabrication of composite STM probes
}

Andrei M. Ionov ${ }^{\mathrm{a}, \mathrm{b}}$, Sergey V. Chekmazova, ${ }^{\mathrm{a},}$, Victor Usov ${ }^{\mathrm{c}}$, Maria E. Nesterova ${ }^{\mathrm{a}}$, Alexander S. Aronin $^{\mathrm{a}, \mathrm{b}, \mathrm{d}}$, Valery N. Semenov ${ }^{\mathrm{a}}$, Igor V. Shvets ${ }^{\mathrm{c}}$, and Sergey I. Bozhko ${ }^{\mathrm{a}, \mathrm{c}, *}$

${ }^{a}$ Institute of Solid State Physics, Russian Academy of Sciences, Chernogolovka, Moscow district 142432, Russia

${ }^{\mathrm{b}}$ National Research University Higher School of Economics, Moscow 101000, Russia

' School of Physics, Centre for Research on Adaptive Nanostructures and Nanodevices (CRANN), Trinity College Dublin, Dublin 2, Ireland

${ }^{\mathrm{d}}$ National University of Science and Technology MISiS, Moscow, Russia

* Corresponding authors at: Institute of Solid State Physics, Russian Academy of Sciences, Chernogolovka, Moscow district 142432, Russia.

E-mail addresses: chekmazov@issp.ac.ru (S.V. Chekmazov), bozhko@issp.ac.ru (S.I. Bozhko). 


\begin{abstract}
Fracturing microscale constrictions in metallic wires, such as tungsten, platinum, or platinum-iridium, is a common fabrication method used to produce atomically sharp tips for scanning tunneling microscopy (STM), field-emission microscopy and field ion microscopy. Typically, a commercial polycrystalline drawn wire is locally thinned and then fractured by means of a dislocation slip inside the constriction. We examine a special case where a dislocation-free microscale constriction is created and fractured in a single crystal tungsten rod with a long side parallel to the [100] direction. In the absence of dislocations, vacancies become the main defects in the constriction which breaks under the tensile stress of approximately $10 \mathrm{GPa}$, which is close to the theoretical fracture strength for an ideal monocrystalline tungsten. We propose that the vacancies are removed early in the tensile test by means of deformation annealing, creating a defect-free tungsten constriction which cleaves along the $\mathrm{W}(100)$ plane. This approach enables fabrication of new composite STM probes which demonstrate excellent stability, atomic resolution and magnetic contrast that cannot be attained using conventional methods.
\end{abstract}

Keywords: Scanning tunneling microscopy (STM), STM probe preparation, Tension test, Vacancies, Fracture mechanism, Spinsensitive composite probe. 


\section{Introduction}

Scanning tunneling microscopy (STM) is a powerful experimental technique for direct visualization of surface morphology, electronic structure and magnetic properties with atomic scale resolution. The sensitivity, resolution and other important characteristics of STM largely depend on its key component; the sharp conductive probe, mainly fabricated by electrochemical etching of commercial drawn polycrystalline tungsten wire or sometimes a single crystalline W rod [1-8]. The tips are produced by plastic deformation and fracture of nanoscale constrictions formed by etching at the surface of electrolyte [5,7,9-14]. This technique allows for the fabrication of STM probes with a tip apex radius of less than $30 \mathrm{~nm}$ enabling STM imaging with atomic and subatomic resolution [3,4,6,8]. Although the method has been widely used, a detailed quantitative study of the process is still required. Mainly it is due to the use of commercial tungsten wire with unknown and uncontrolled density of defects and orientation of the $\mathrm{W}$ grain at the tip apex. Studying deformation and fracturing in single crystalline metallic constrictions can help to better understand the STM probe fabrication process and how it can be controlled or altered by changing the density of defects. In particular, at what stage of the thinning process can the constriction material be considered to be a perfect crystal. Growth methods for perfect crystalline materials have been developed only for a limited class of solids such as silicon [15] while most materials contain defects in their crystal structure. Lattice defects for example, such as grain boundaries, dislocations and vacancies determine mechanisms of deformation and breaking and significantly affect results of deformation and fracture experiments [16]. In the case of metals, the issue is addressed by using reduced size crystals, nanowires, whiskers or nanopillars [17-21]. For example, the in situ compressive and tensile testing was conducted along the long axis of tungsten nanopillars, milled by focused ion beam, where deformation proceeded in a nonuniform manner along the [001] direction [22,23]. In particular, the plastic deformation section of the stress-strain curve demonstrated steps corresponding to discrete stages of stress reductions with increasing strain. The tension experiments reveal prominent size effects while in compression the effect is relatively small [22].

Besides the fundamental interest in deformation and fracturing of single crystalline tungsten, a microscale atomically flat apex terminating a tungsten STM tip is a unique crystallographically oriented substrate of high surface energy, where a wide variety of materials can be epitaxially grown. This provides an opportunity for the fabrication of unique composite probes, of which production is either undeveloped or not feasible by conventional mechanical or electrochemical methods. Using this approach, the probe's functional parameters such as conductivity, band gap, magnetic moment or easy axis can be customized for the investigation of particular samples or to meet specific experimental requirements. In this regard, magnetite is a promising conductive 
oxide for spin-dependent STM due to its sensitivity to the surface magnetic moment and local density of states (LDOS) [24-27]. Bulk magnetite undergoes the Verwey transition at $T_{\mathrm{v}} \approx 120 \mathrm{~K}$ from a high temperature "bad metal" conducting phase to a low-temperature insulating phase. However, magnetite nanocrystals and single crystal thin films exhibit an electrically driven phase transition below the Verwey temperature. The signature of this transition is the onset of sharp conductance switching in high electric fields, which is hysteretic in voltage similar to memristor switching [28]. Epitaxial growth of magnetite on the flat tip apex provides an opportunity to study memristor switching due to electric field driven Verwey transition in STM experiments. In this work we show that tungsten single crystal probes can be functionalized by the epitaxial growth of a magnetite $\left(\mathrm{Fe}_{3} \mathrm{O}_{4}\right)$ nanoparticle on top of a flat $\mathrm{W}(100)$ apex and demonstrate the effectiveness and functionality of these probes.

\section{Material and methods}

Figure 1 shows a photograph of an experimental setup used to study the deformation and fracture mechanisms of the constrictions. A Pt ring was attached to the electromechanical universal testing machine (Instron ${ }^{\circledR}$ ) and placed between two opposing grips. Tungsten single crystal rods were passed through the center of the ring and gripped at both ends such that a long side coincided with the direction of tensile force. The $2 \mathrm{M} \mathrm{NaOH}$ electrolyte was suspended in the ring using a pipette. The electrochemical reaction was initiated by applying a potential difference between the tungsten rod and platinum ring which served as anode and cathode, respectively. As the tungsten rod was electrochemically etched, the tensile stress was applied by a mechanical test machine thus allowing simultaneous mechanical tensile testing and electrochemical etching. This proved to be particularly important as it was shown later that etching can remove surface defects such as microcracks or sharp shears formed in the pulling process. Initially, electrochemical etching was conducted alone in order to create a constriction in a tungsten rod, followed by the activation of tensile deformation produced by uniaxial stretching applied at a constant rate of $5 \mu \mathrm{m} / \mathrm{min}$. 


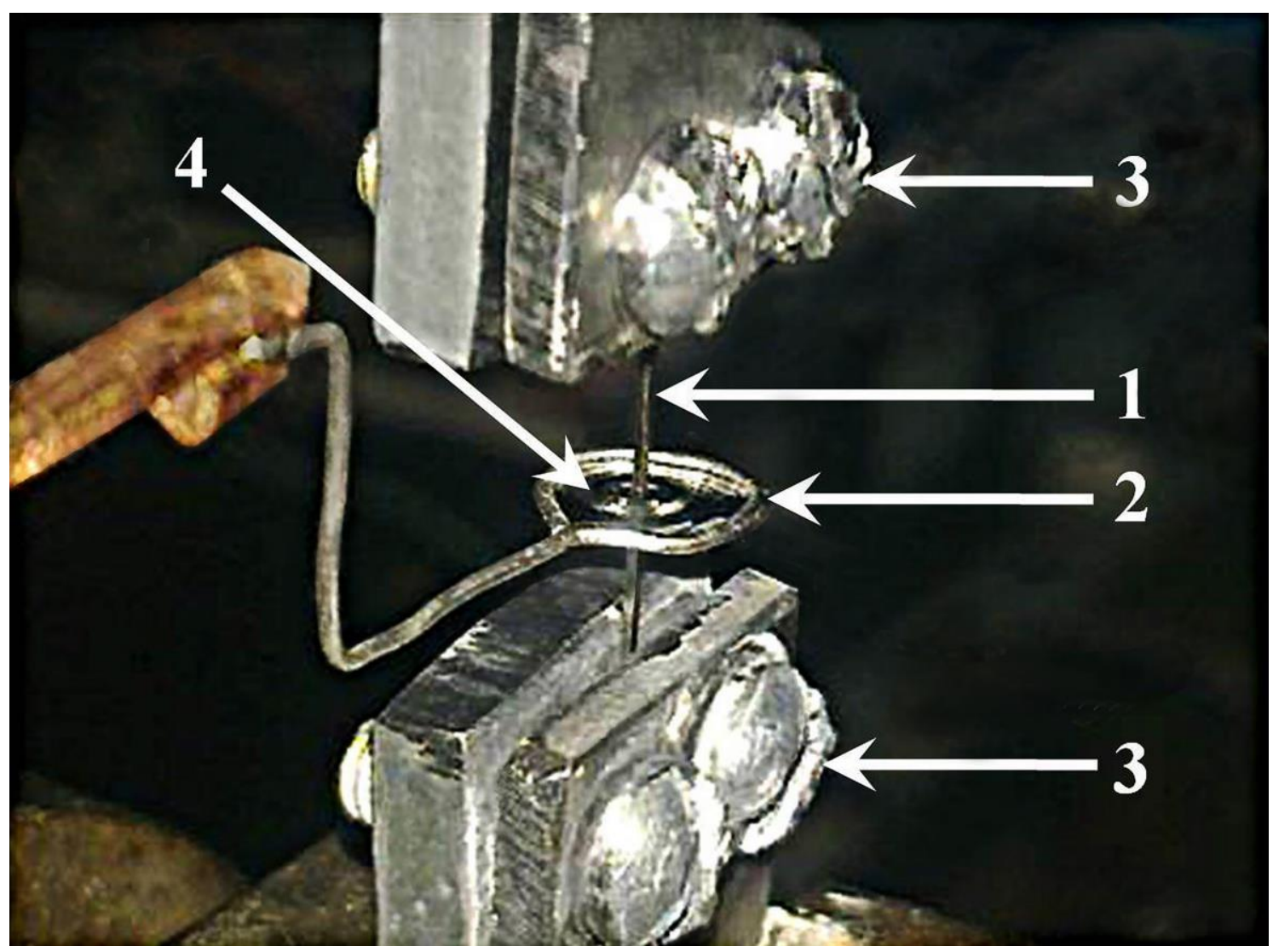

Fig. 1. Photograph of an electrochemical cell set up on the electromechanical universal testing machine (Instron ${ }^{\circledR}$ ): $1-\mathrm{W}$ single crystalline sample; 2 - Pt ring; 3 - metal grips; $4-2 \mathrm{M} \mathrm{NaOH}$ electrolyte film.

A modified method for creating tungsten STM probes using electrochemical etching was used in this work, as shown in Fig. 2. A stainless steel cylindrical cathode was placed in a beaker with a $2 \mathrm{M}$ solution of $\mathrm{NaOH}$. Single crystal tungsten rectangular rods, with their long axes along the [100] crystallographic direction, served as anodes and were placed coaxially with the cylindrical cathode. The rods were prepared from $99.95 \%$ purity bulk single crystal tungsten, grown using an electron-beam floating zone with the growth axis along the [100] direction $[29,30]$. The dislocation density in the crystals was around $10^{5} \mathrm{~cm}^{-2}$ corresponding to an average inter-dislocation distance of $\approx 30 \mu \mathrm{m}$. The $0.5 \times 0.5 \times 15 \mathrm{~mm}^{3}$ rectangular rods were cut from the tungsten single crystal by electro-sparking, with an orientation accuracy of $\sim 3^{\circ} .100 \mu \mathrm{m}$ deep microcracks were removed from the surface by mechanical grinding after being created by high mechanical stress in the spark region. The rods were electrochemically polished in $2 \mathrm{M}$ aqueous solution of $\mathrm{NaOH}$ for several seconds. Finally, the rods were electrochemically etched in a $2 \mathrm{M}$ solution of $\mathrm{NaOH}$ by oxidative dissolution at a potential of $3.8-4.1 \mathrm{~V}$. The immersed part of the rod (1 - $3.5 \mathrm{~mm}$ long) was protected from etching by a polymer layer (epoxy, varnish or Teflon $^{\mathrm{TM}}$ ) resulting in the formation of a constriction at the solution surface due to a capillary wetting effect (see Fig. 2). As the etching progressed, the constriction gradually reduced in diameter until it fractured under the weight of the suspended section, when the breaking tensile strength of tungsten was exceeded. 


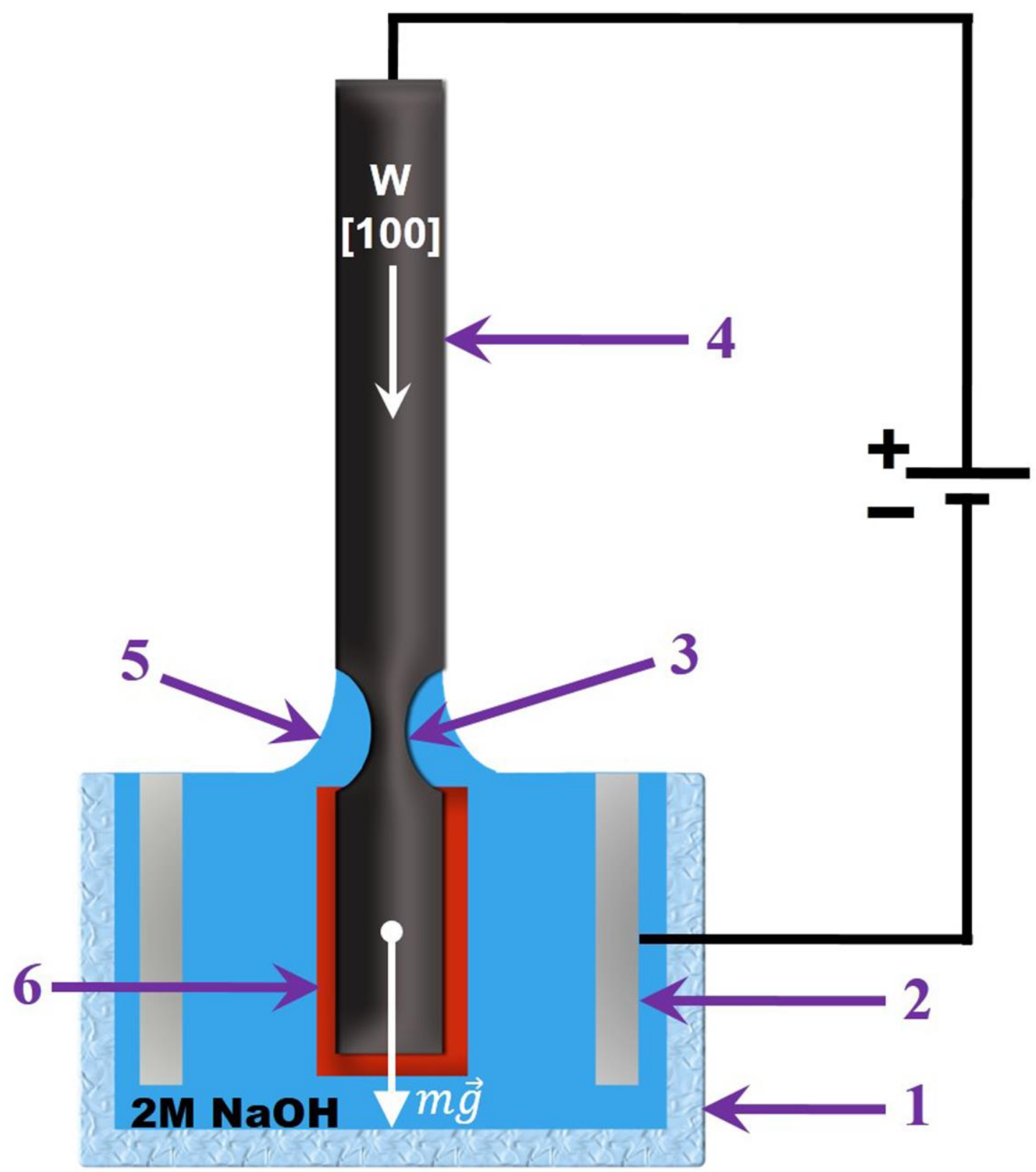

Fig. 2. Schematic of the tungsten probes preparation: 1 - glass beaker; 2 - cylindrical stainless steel cathode; 3 - sample constriction; 4 -W single crystalline rod; 5 - meniscus; 6 - protective coverage (epoxy, varnish or Teflon ${ }^{\mathrm{TM}}$ ).

The fracture point (the area of the apex flat) was controlled by varying weight of the immersed part, which was proportional to its length. Commercial drawn polycrystalline tungsten wire 0.3 $\mathrm{mm}$ in diameter was also used in experiments for comparison.

\section{Results and Discussion}

Deformation of the constriction before fracture is most commonly realized in metals under conditions of plastic deformation, which is connected to the motion of dislocations [16]. As was mentioned above, the average distance between dislocations in our tungsten crystals is approximately $30 \mu \mathrm{m}[29,30]$. When the cross-sectional dimensions of a constriction are smaller 
than the mean inter-dislocation distance, it is highly likely that only point defects are present in the constriction volume, and plastic deformation proceeds by mechanism other than motion of linear defects. Therefore, the size of the deformation region is the key factor defining the plastic deformation and fracturing mechanism.

Figure 3(a) shows an SEM image of a tungsten rod terminated with a flat W(100) surface created by fracturing a single crystal rod in a constricted area. It must be noted that the rods were deliberately cut with a long axis along the [100] direction, as the cleavage $\{100\}$ and twinning $\{112\}$ planes are the most probable planes for brittle fractures in the $b c c$ tungsten lattice $[19,31]$. The fractured area was easily identified as a flat apex and was measured to be approximately 35 $\mu \mathrm{m}$ across (Fig. 3(a)). The boundary of the plastic deformation region was identified from the shape of the apex cone and is marked by a yellow dotted line in Fig. 3(a). The constriction diameter measured approximately $59 \mu \mathrm{m}$ across the boundary of the deformation region. The length of the deformation region from the apex to its supposed boundary (marked by yellow dotted line) was approximately $20-25 \mu \mathrm{m}$. Considering that measurements were conducted only on one half of the rod, the total length of the deformation region doubles and can be estimated as $40-50 \mu \mathrm{m}$. Notably, the length of the plastic deformation region is comparable with the diameter of the constriction and the average distance between dislocations. Experimental tensile stress-strain curves were plotted as a function of the rod strain $\varepsilon$ defined as $\varepsilon=\frac{\Delta l}{l}$, where $\Delta l$ is the constriction elongation and $l$ is the length of the deformation region, which cannot be accurately directly measured. However, it was demonstrated in several experiments on the fracture of rod constrictions that the length of the plastic deformation region was approximately the same as the constriction diameter in the fractured area. The estimated constriction elongation under deformation gives a value of $\varepsilon$ of about $180 \%$ (Fig. 3(b)), which agrees with earlier macroscopic deformation studies [32,33]. However this value should be regarded as an estimate, due to the variation of cross-sectional area in the deformation region resulting from the elongation and electrochemical reaction.
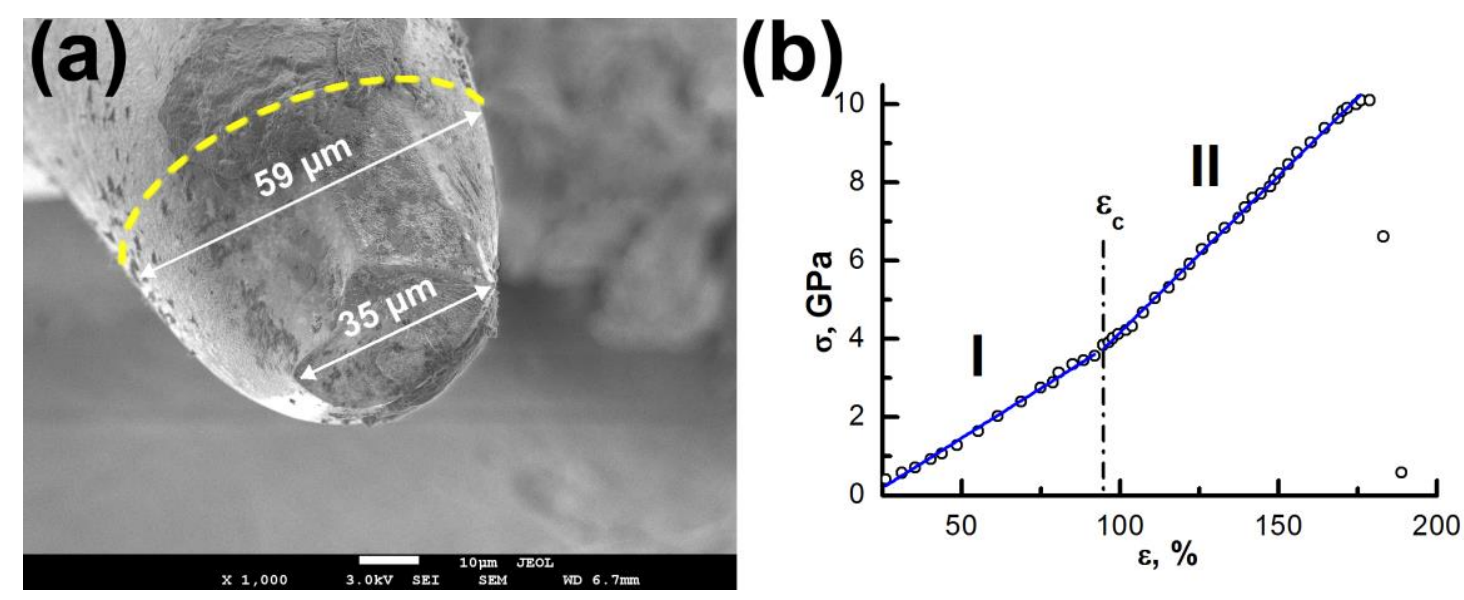
Fig. 3. (a) SEM image of a tungsten single crystal rod fractured in the constricted area by a tensile stress applied along the [100] direction while being electrochemically etched in order to remove the surface defects created in the deformation process, (b) corresponding tensile stress-strain curve. Regions I and II correspond to different linear sections of the graph and attributed to different deformation mechanisms.

Figure 3(b) shows a tensile stress-strain curve obtained with the in situ electrochemical etching. Regions I and II corresponding to different mechanisms of deformation can be clearly distinguished (as will be further discussed). The stress-strain dependence in both regions is linear indicating that the deformation proceeds by different mechanism than movement and multiplication of dislocations. Notably, the deformation and a subsequent fracture proceed without a characteristic plastic deformation region typical for metals (normally caused by dislocation movement). The fracture strength $\sigma_{\mathrm{m}}=10 \mathrm{GPa}$ is also very high compared to, for example, 1.26 GPa measured in other studies [32,33] (see Fig. 3(b)) and is close to the theoretical value for an ideal tungsten crystal [17,34-38].

Figure 4 demonstrates the dramatic effect of electrochemical etching on the deformation and breaking process. It must be emphasized that electrochemical etching did not result in any significant changes in the constriction diameter throughout the entire experiment. Changes in the constriction diameter due to the etching were estimated as $1 \%$ or less based on the anode current measurements. In order to study the effects of etching on the deformation and fracture, we repeated the uniaxial tensile test on identical constrictions under the same conditions, except that in some of the tests electrochemical etching was not activated (a potential difference between cathode and anode was not applied). We also investigated the effects of etching on the deformation and breaking of constrictions created in a commercial drawn polycrystalline tungsten wire, which is the commonly used source material in the point probe fabrication. Essentially, these experiments showed that electrochemical etching affects deformation process only in single crystal dislocation-free constrictions and indicated the important role of bulk defects in the near-surface region. Figure 4 shows stress-strain curves recorded for single crystal constrictions with the uniaxial tensile stress applied along the [100] direction in the absence (red curve and presence (black curve) of electrochemical etching. Initially, the deformation process in the monocrystalline rods is not affected by electrochemical etching and both curves follow approximately the same path until a tensile stress $\sigma \approx 3 \mathrm{GPa}$ is reached where the constriction, stretched without etching, enters a regime of plastic deformation and breaks. The etched constriction remains intact at this point, but the corresponding stress-strain curve abruptly changes to a higher slope at a kink point at $\varepsilon_{\mathrm{c}}$, indicating a transition to a different deformation regime (switching from regime I to regime II in Fig. 4). After this transition, the constriction further elongates under much higher strain $\varepsilon$ until it breaks when the tensile stress reaches the fracture point at $\sigma_{\mathrm{m}}=10 \mathrm{GPa}$. Essentially, activation of electrochemical etching approximately 
doubles the strain and triples the stress at the fracture point. A significantly different stress-strain curve, shown in blue in Fig. 4, was recorded for a constriction created in a commercial drawn polycrystalline wire under the etching conditions (as discussed later). Note, the single crystal constriction fracture at significantly higher strain and stress than polycrystalline.

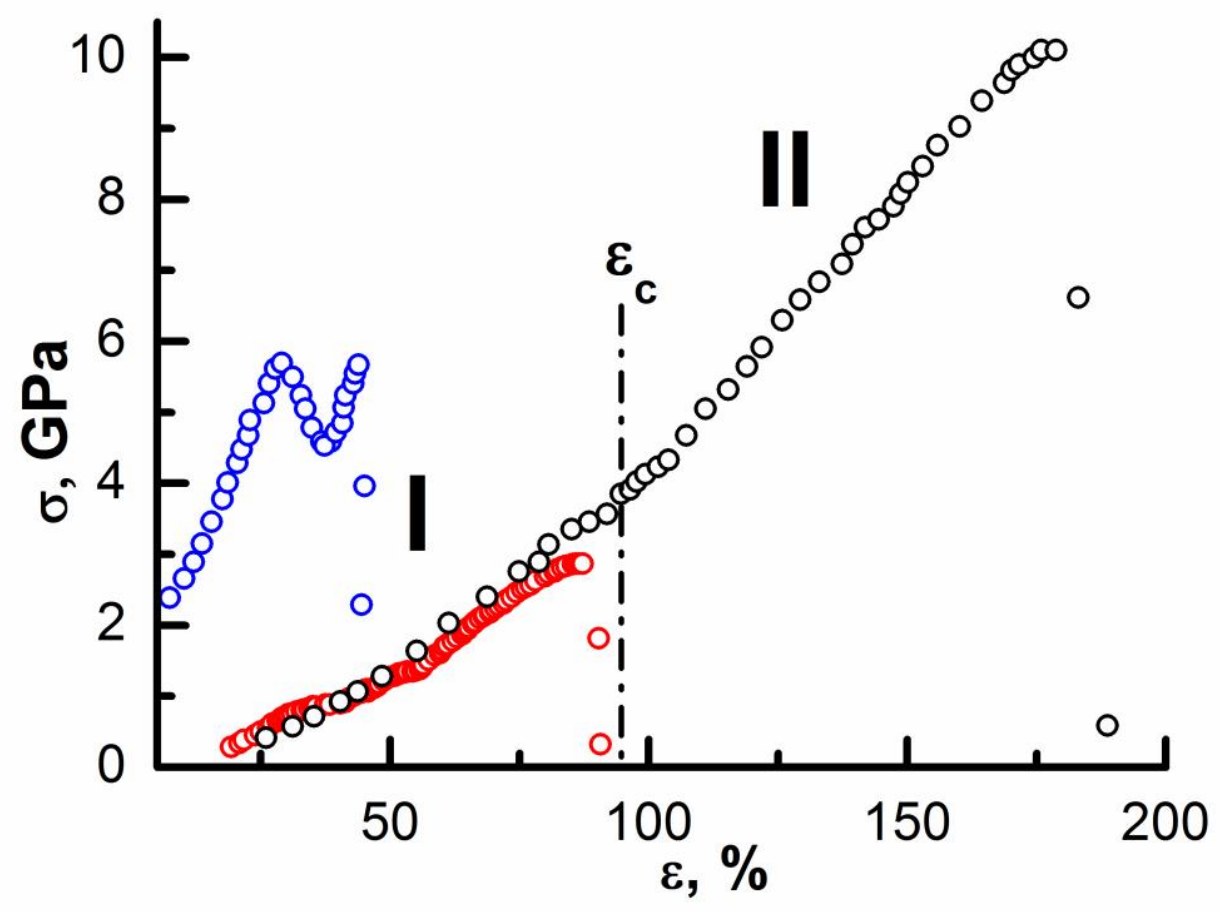

Fig. 4. Tensile stress-strain curves of mono- and polycrystalline tungsten constriction: blue curve corresponds to a tensile test conducted with electrochemical etching of a commercial drawn polycrystalline tungsten wire; black and red curves were recorded in a tensile tests on single crystal samples in the presence and absence of electrochemical etching, respectively.

Figure 5(a) shows an SEM image of a fractured constriction of single crystal tungsten. Constrictions with a diameter larger than $50 \mu \mathrm{m}$ fractured through the defect assisted mechanism creating rough fractured surfaces (Fig. 5(a)) in contrast to the smooth termination on fractures created for constrictions with a diameter less than $50 \mu \mathrm{m}$ (Fig. 3(a)). This would indicate that fracturing proceeds by different mechanisms for diameters greater and smaller than the mean inter-dislocation distance, which is manifested in terms of surface roughness. Figure 5(b) shows an SEM image of a constriction created in a commercial drawn polycrystalline tungsten wire. Although the constriction diameter of the commercial drawn polycrystalline wire is only $24 \mu \mathrm{m}$ across (less than $35 \mu \mathrm{m}$ for the single crystal constriction in Fig. 3(a)), it still contains a number of defects (e.g. dislocations) and fails by defect assisted mechanism creating a rough fractured surface. 


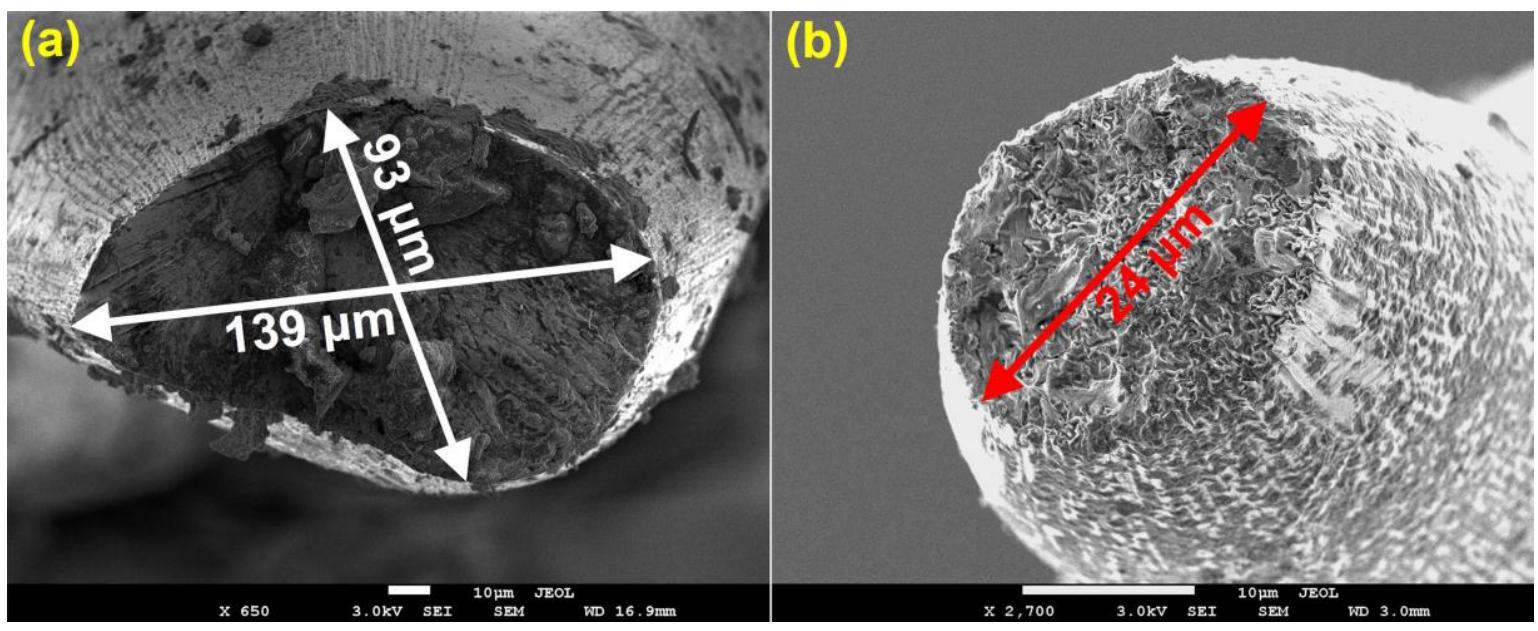

Fig. 5. SEM images of $\mathrm{W}$ surfaces in the constriction region: (a) surface of a fractured constriction created in a tungsten single crystal rod pulled along the [100] direction in the absence of the electrochemical etching. The fractured area is approximately $139 \times 93 \mu \mathrm{m}^{2}$; (b) surface of a fractured constriction created in commercial drawn polycrystalline tungsten wire stretched by simultaneous uniaxial tension and electrochemical etching. The fractured area is approximately $24 \mu \mathrm{m}$. Both surfaces display rough termination.

These results indicate that in the case of simultaneous uniaxial tension and etching a different mechanism of deformation, taking into account the role of point defects must be considered. There is limited data on mechanical fracturing of small size tungsten crystals assisted by simultaneous electrochemical etching. At the same time, changing a material's bulk mechanical properties through modification of a surface layer is a well-known and commonly used technique in materials science. For example, $\mathrm{NaCl}$ crystals demonstrated higher plasticity and strength when their mechanical properties were tested in water, as compared to air, due to continuous dissolution of surface material [39-42]. Brittle fractures in crystals start by nucleation of surface defects such as microcracks or sharp shifts [43] and removal of these defects by dissolution blocked the microcrack growth or reduced the microcrack propagation speed into the bulk. As a result, the breaking strength of the rock salt of approximately $1.6 \mathrm{GPa}$ was achieved $[39,41,42]$, close to the theoretical strength of $2 \mathrm{GPa}$ [44].

Deformation and fracture of perfect dislocation-free crystals depends on a density of point defects in the material. For example, annealing polycrystalline $\mathrm{Cu}$ with a grain size of $200 \mathrm{~nm}$ or less at $200{ }^{\circ} \mathrm{C}$ or higher results in a $10-20 \%$ change of the Young's modulus due to the change in the density of vacancies in the $\mathrm{Cu}$ grains [45]. At equilibrium, the density of intrinsic point defects in a single crystal is proportional to $\exp \left(-\frac{E}{k_{\mathrm{B}} T}\right)$, where $E$ is the energy of a point defect formation, $T$ is the temperature of crystal formation (or melting point) and $k_{\mathrm{B}}$ is the Boltzmann constant. At pre-melting temperatures, the dominant point defects in tungsten are vacancies. Since the melting point for tungsten is very high $(T=3695 \mathrm{~K})$, the density of vacancies can be as high as $3.4 \%[31,46]$. They formed at the interface between the liquid molt and the crystal surface and cannot be avoided by optimizing the growth conditions. When the sample is cooling down during crystal growth the vacancy concentration reduces, tracking the equilibrium value, 
so long as the vacancy relaxation time remains short enough. However, when the vacancy relaxation time exceeds the cooling time, the concentration of vacancies can no longer reach the equilibrium value at any given moment. The relaxation time decreased exponentially with temperature and becomes comparable with the crystal growth time at $2000 \mathrm{~K}$ (approximately 1 hour in our case) [47]. At $T=2000 \mathrm{~K}$ the equilibrium concentration of vacancies is still high at about $0.001 \%$ [46,47]. As a result, even a small dislocation-free crystalline constriction would still contain a nonequilibrium high density of vacancies. Under the tensile stress $\sigma$, vacancies come into motion similar to the Nabarro-Herring mechanism $[48,49]$, and the flux of vacancies depends exponentially on the applied stress [16,49]. However, different to the Nabarro-Herring creep, they do not nucleate on the constriction surface. Instead they drift towards the surface from the bulk of constriction (Fig. 6). Under tensile force, the stress applied to the constriction region is significantly higher than applied to the rest of the rod (due to a smaller size of the constriction's cross-sectional area) which results in a significantly higher vacancy flux in the constriction volume (Fig. 6). As a result, even at a low tensile load, the constricted region becomes depleted of vacancies and the further deformation proceeds on a virtually defect-free crystal with a perfect lattice (Fig. 3(b)). We propose that this change in the constriction stoichiometry manifests itself as a slope rise of the stress-strain curve at $\varepsilon_{\mathrm{c}}$ which divides the deformation curve in two regions. In the region I, corresponding to the initial deformation, density of vacancies is significantly higher than in the region II, where tungsten lattice is depleted of point defects. The slope rise indicates depletion of the point defects from the bulk of the constriction by means of deformation annealing process.

Figure 6 schematically shows the migration of vacancies under the influence of an external tensile stress. Vacancies migrate towards the constriction surface, where they participate in the nucleation of microcracks, which if developed would result in a significant strength reduction. However, when a tungsten constriction is simultaneously subjected to tensile deformation and electrochemical etching the surface layer is constantly removed. Thus cracks are prevented from propagating in the same manner as the dissolution of the surface layer in $\mathrm{NaCl}$ crystal which prevents it from early fracture [39-42]. As a result, the experimentally obtained ultimate tensile strength $\sigma_{\mathrm{m}}=10 \mathrm{GPa}$ is close to its lower theoretical limit ranging between $14.4 \mathrm{GPa}$ [17,38] and $30 \mathrm{GPa}$ [34-38]. Nevertheless, the experimentally obtained ultimate tensile strength is still lower than the theoretical limit, which can be explained by a large surface-to-volume ratio in the smallsize constrictions: the lower atomic coordination on the surface results in the lower fracture strength of a surface layer thus leading to an earlier failure of the constriction. Also, the tension axis is not precisely aligned to the [100] direction due to the uncertainty in the alignment of the 
rod's long axis to crystallographic direction. This can result in a shear stress component $\tau$ normal to the [100] direction in the system.
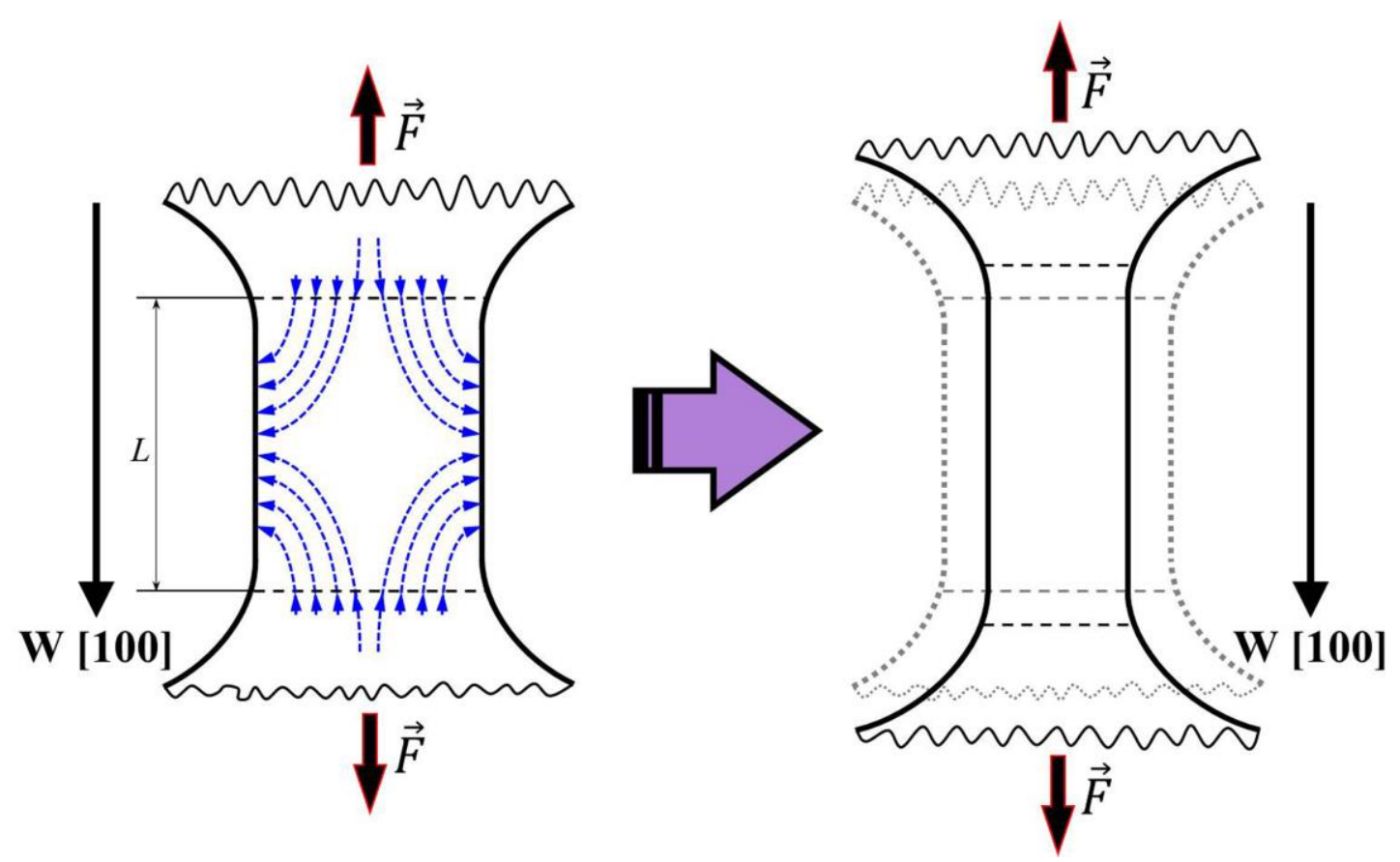

Fig. 6. Schematic of mass vacancy drift (shown with blue dotted arrows) in the constriction region under uniaxial tensile stress applied in the [100] direction resulting in a macroscopic change in the form of a constriction of tungsten rod.

While the simultaneous electrochemical etching and tensile deformation of the dislocationfree tungsten constrictions results in a significant enhancement of the fracture strength, it can also have an adverse effect on the bulk size crystals inevitably containing dislocation networks [32,33]. For example, deformation of macroscopic monocrystalline tungsten results in the formation of approximately $80 \mu \mathrm{m}$ thick pre-surface layer, with a dislocation density higher than in the crystal bulk [32]. The breaking strength of this layer is higher than of the bulk due to the strain hardening effect in the region of a higher dislocation density. Electrochemical etching of a bulk tungsten crystal during deformation continuously removes the dislocation-rich layer, thus increasing the dislocation mobility and decreasing the ultimate tensile strength [32,33]. For example, the ultimate tensile strength for the macroscopic crystalline tungsten is reduced by a factor of 1.5 from 1.26 GPa when a tensile test was combined with electrochemical etching [33].

Tensile deformation assisted by electrochemical etching was used to develop a novel two step fabrication method for unique composite STM probes. First, tungsten tips were created by electrochemical etching with an apex terminated by the (100) plane (Fig. 2) and then a magnetite nanoparticle was epitaxially grown of on top of the apex. Magnetite was chosen as the working end for the probes due to the spin-dependent tunneling previously observed in the STM studies of magnetite [24-26]. Crystallographic structure of $\mathrm{Fe}_{3} \mathrm{O}_{4}$ comprises two $\mathrm{Fe}$ sublattices $(\mathrm{Fe}(A)$ and $\operatorname{Fe}(B)$ ) with the magnetic moments ferrimagnetically aligned. It is known from density 
functional theory calculations [50] and spin resolved photoelectron spectroscopy [51] that the density of states (DOS) for the $\operatorname{Fe}(A)$ and $\operatorname{Fe}(B)$ sublattices is significantly different. Since tunneling current in STM is proportional to the LDOS, depending on the bias voltage tunneling current can be dominated by electrons localized on either $\operatorname{Fe}(A)$ or $\operatorname{Fe}(B)$ sublattice. Therefore, the composite tungsten probes terminated by a magnetite nanoparticle can be a solution for consistent and reliable spin-polarized STM imaging.

STM imaging capabilities of the composite probes were demonstrated under UHV conditions ( $p=2 \times 10^{-11}$ Torr) at cryogenic temperatures $(T=78 \mathrm{~K})$. A 0.2 monolayer thick Fe film was deposited on the $\mathrm{MoO}_{2} / \mathrm{Mo}(110)$ surface. Figure 7 shows STM images of the same area of the surface recorded with the composite $\mathrm{Fe}_{3} \mathrm{O}_{4} / \mathrm{W}(100)$ probe at different values of bias. No drift corrections were applied during scanning, therefore images are slightly shifted. Both STM images in Fig. 7 revealed the $2.3-2.4 \mathrm{~nm}$ wide $\mathrm{MoO}_{2}$ nanorows in accordance with previous

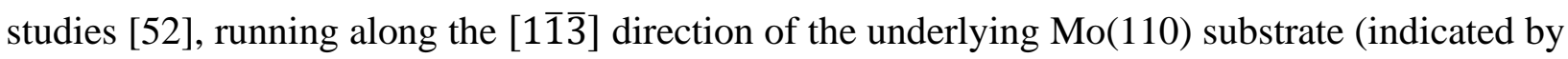
long red arrows). $\mathrm{Fe}$ atoms on the $\mathrm{MoO}_{2} / \mathrm{Mo}(110)$ surface formed small nanoclusters with the size of several $\mathrm{Fe}$ atoms across as in previous studies [27]. Individual surface atoms (indicated by green circles in Fig. 7) were resolved at different bias $U_{\text {bias }}$ demonstrating atomic resolution capabilities of the composite probes.
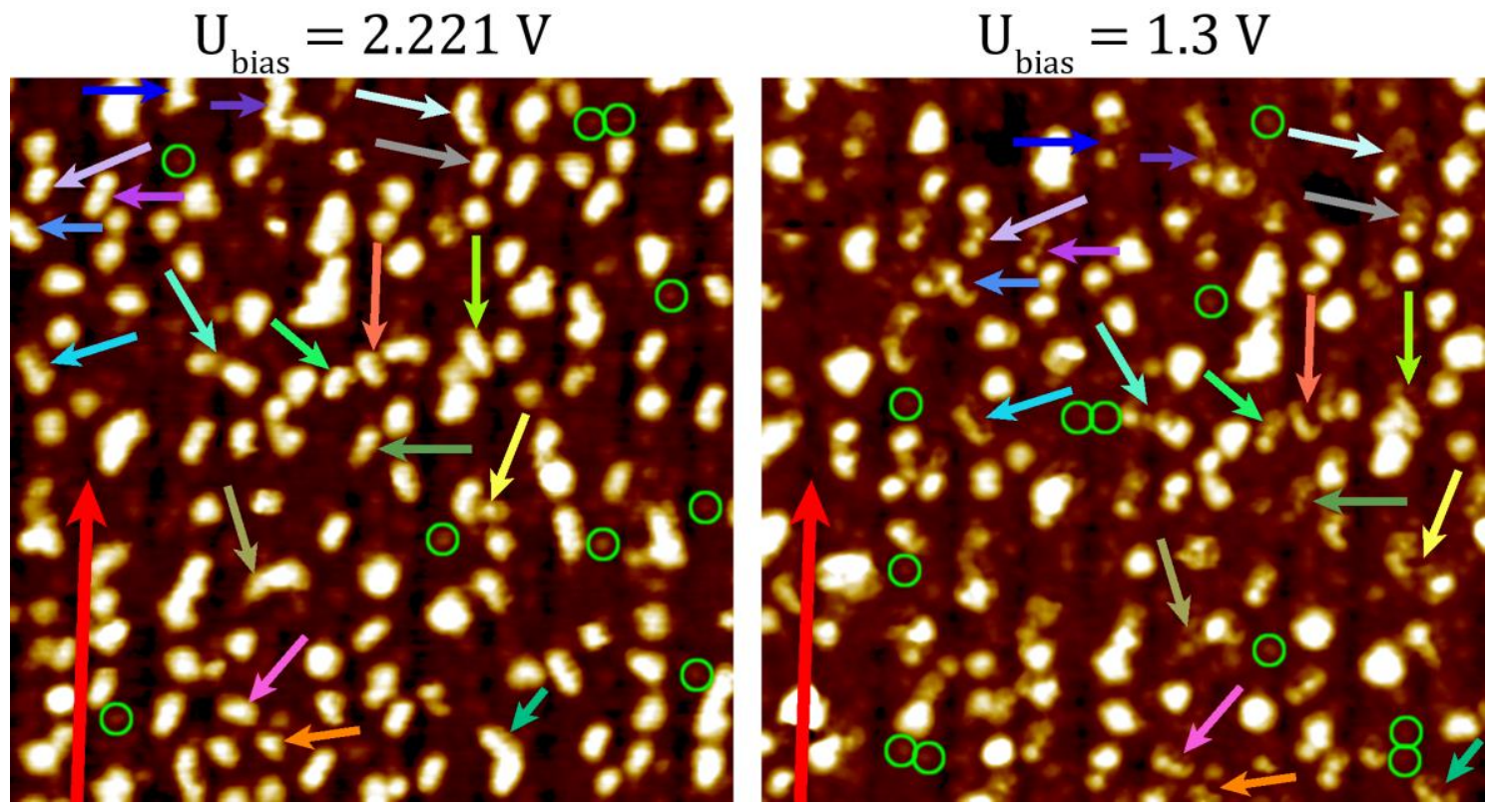

Fig. 7. STM images of the same $26.2 \times 26.2 \mathrm{~nm}^{2}$ area of $\mathrm{Fe}$ nanoclusters on the $\mathrm{MoO}_{2} / \mathrm{Mo}(110)$ surface, recorded with a composite probe at different values of bias voltage: $U_{\text {bias }}=2.221 \mathrm{~V}$ (left) and $U_{\text {bias }}=1.3 \mathrm{~V}$ (right). Tunneling current remained at the same value $I_{\text {tun }}=400 \mathrm{pA}$. Long red arrows indicate nanorows on the $\mathrm{MoO}_{2} / \mathrm{Mo}(110)$ surface, which are aligned along the

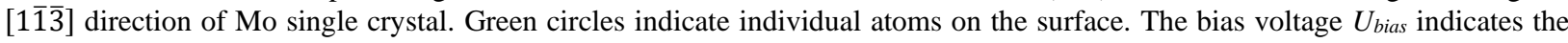
sample bias with respect to the probe.

Figure 7 clearly shows that while some nanoclusters appear the same under different bias, others have reduced their contrast or change their contrast completely from positive to negative. This indicates that the tunneling current is suppressed at $U_{\text {bias }}=1.3 \mathrm{~V}$ for clusters with certain 
orientation of magnetic moment. The clusters that changed or altered their contrast upon the change of bias are indicated by arrows of the same color in the STM images. The $\mathrm{MoO}_{2} / \mathrm{Mo}(110)$ surface topography is not homogeneous due to the presence of nanorows and the surface molecular field can be different for nanoclusters at different locations. It is likely that this molecular field is responsible for a fixed magnetic moment of nanoclusters that showed different contrast at different bias. Apparently, the influence of this field on magnetic moment of other Fe nanoclusters was weaker as they demonstrated superparamagnetic behavior in the spin-sensitive STM measurement. Earlier superparamagnetic behavior of Fe nanoclusters on the $\mathrm{MoO}_{2} / \mathrm{Mo}(110)$ surface was observed in [53].

\section{Conclusions}

We studied deformation and fracture in tungsten microscale defect-free crystals. Single crystal constrictions with a mean diameter less than a mean inter-dislocation distance were created in a tungsten rod and subjected to the simultaneous electrochemical etching and tensile test which revealed the stress-strain curves made of two linear segments of different slope (Fig. 3(b)). Counterintuitively, the slope was lower for the initial stage of deformation than for the later stage where the strain was significantly higher. Activation of electrochemical etching during deformation test approximately doubled the strain and tripled the stress at the fracture point. We suggest that, in the absence of dislocations in the constriction region, the rod deformation and fracture were accompanied by migration of the nonequilibrium point defects (namely vacancies) which are abundant in tungsten single crystal. The slope rise indicated depletion of the point defects from the bulk of the constriction by means of the deformation annealing process, namely migration of vacancies from the bulk under the uniaxial tensile stress and their condensation on the surface. The surface layer containing vacancies was constantly etched away thus preventing nucleation and propagation of defects into the bulk. As a result, the defect-free constrictions were created and fractured under the stress of up to $10 \mathrm{GPa}$ which is relatively close to the theoretical fracture strength of tungsten.

A novel and simple method was developed for fabrication of spin-sensitive composite probes for simultaneous topographic and magnetic STM imaging. A microscale constriction was electrochemically etched in a crystalline tungsten rod and broken along the $\mathrm{W}(100)$ plane by a tensile pull creating an atomically flat apex where a magnetite nanoparticle was epitaxially grown. The probes demonstrated excellent stability, atomic resolution and magnetic contrast in the STM measurements conducted on Fe nanoclusters grown on the $\mathrm{MoO}_{2} / \mathrm{Mo}(110)$ substrate.

\section{Acknowledgments}


Authors thank V. M. Chernyak for technical support of experiments. We acknowledge support of the Research Facility Center at the ISSP of RAS in mechanical testing.

\section{Funding}

The work was partially supported by RFBR Grant 19-29-03021. Financial support of the state assignment of the Institute of Solid State Physics of Russian Academy of Sciences is gratefully acknowledged.

\section{References}

[1] H.-W. Fink, Mono-atomic tips for scanning tunneling microscopy, IBM J. Res. Dev. 30 (1986) 460-465. http://dx.doi.org/10.1147/rd.305.0460.

[2] D.K. Biegelsen, F.A. Ponce, J.C. Tramontana, Simple ion milling preparation of <111> tungsten tips, Appl. Phys. Lett. 54 (1989) 1223-1225. http://dx.doi.org/10.1063/1.100722.

[3] A.N. Chaika, S.S. Nazin, V.N. Semenov, S.I. Bozhko, O. Lübben, S.A. Krasnikov, K. Radican, I.V. Shvets, Selecting the tip electron orbital for scanning tunneling microscopy imaging with sub-ångström lateral resolution, Europhys. Lett. 92 (2010) 46003. http://doi.org/10.1209/0295-5075/92/46003.

[4] A.N. Chaika, S.S. Nazin, V.N. Semenov, N.N. Orlova, S.I. Bozhko, O. Lübben, S.A. Krasnikov, K. Radican, I.V. Shvets, High resolution STM imaging with oriented single crystalline tips, Appl. Surf. Sci. 267 (2013) 219223. https://doi.org/10.1016/j.apsusc.2012.10.171.

[5] A.N. Chaika, N.N. Orlova, V.N. Semenov, E.Yu. Postnova, S.A. Krasnikov, M.G. Lazarev, S.V. Chekmazov, V.Yu. Aristov, V.G. Glebovsky, S.I. Bozhko, I.V. Shvets, Fabrication of [001]-oriented tungsten tips for high resolution scanning tunneling microscopy, Sci. Rep. 4 (2014) 3742. https://doi.org/10.1038/srep03742.

[6] A.N. Chaika, Visualization of Electron Orbitals in Scanning Tunneling Microscopy, JETP Lett. 99 (2014) 731741. https://doi.org/10.1134/S0021364014120054.

[7] W. Paul, P. Grütter, Field Ion Microscopy for the Characterization of Scanning Probes, in: C.S.S.R. Kumar (Ed.), Surface Science Tools for Nanomaterials Characterization, Springer-Verlag Berlin Heidelberg, Heidelberg, 2015, pp. 159-198.

[8] A.N. Chaika, High Resolution STM Imaging, in: C.S.S.R. Kumar (Ed.), Surface Science Tools for Nanomaterials Characterization, Springer-Verlag Berlin Heidelberg, Heidelberg, 2015, pp. 561-619.

[9] J.P. Ibe, P.P. Bey, Jr., S.L. Brandow, R.A. Brizzolara, N.A. Burnham, D.P. DiLella, K.P. Lee, C.R.K. Marrian, R.J. Colton, On the electrochemical etching of tips for scanning tunneling microscopy, J. Vac. Sci. Technol. A 8 (1990) 3570-3575. http://dx.doi.org/10.1116/1.576509. 
[10] A.J. Melmed, The art and science and other aspects of making sharp tips, J. Vac. Sci. Technol. B 9 (1991) 601608. http://dx.doi.org/10.1116/1.585467.

[11] R. Zhang, D.G. Ivey, Preparation of sharp polycrystalline tungsten tips for scanning tunneling microscopy imaging, J. Vac. Sci. Technol. B 14 (1996) 1-10. http://dx.doi.org/10.1116/1.589029.

[12] A.I. Olivia, A. Romero G., J.L. Peña, Electrochemical preparation of tungsten tips for a scanning tunneling microscope, Rev. Sci. Instrum. 67 (1996) 1917-1921. http://dx.doi.org/10.1063/1.1146996.

[13] I. Ekvall, E. Wahlström, D. Claesson, H. Olin, E. Olsson, Preparation and characterization of electrochemically etched W tips for STM, Meas. Sci. Technol. 10 (1999) 11-18. http://doi.org/10.1088/0957-0233/10/1/006.

[14] C.J. Chen, Introduction to Scanning Tunneling Microscopy, second ed., Oxford University Press, New York, 2008, pp. 312-329.

[15] A. Muiznieks, J. Virbulis, A. Lüdge, H. Riemann, N. Werner, Floating Zone Growth of Silicon, in: T. Nishinga,

P. Rudolf (Eds.), Handbook of Crystal Growth. Bulk Crystal Growth: Basic Techniques, Vol. IIA (Basic Technologies), second ed., Elsevier B. V., Amsterdam, 2015, pp. 241-279.

[16] R.W. Cahn, P. Haasen, Physical Metallurgy, Vol. 3, third, revised and enlarged ed., Eisevier Science Publishers B. V., Amsterdam, 1983.

[17] S.S. Brenner, Tensile strength of whiskers, J. Appl. Phys. $27 \quad$ (1956) 1484-1491. http://dx.doi.org/10.1063/1.1722294.

[18] E.M. Nadgorny̌̆, Yu.A. Osip’yan, M.D. Perkas, V.M. Rozenberg, Filamentary crystals with almost the theoretical strength of perfect crystals, Sov. Phys. Uspekhi $2 \quad$ (1959) $282-304$. http://dx.doi.org/10.1070/PU1959v002n02ABEH003124.

[19] G.D. Rieck, Tungsten and its compounds, first ed., Pergamon Press Ltd., Oxford, 1967.

[20] W Fucke, U Seydel, Improved experimental determination of critical-point data for tungsten, High Temp. High Press. 12 (1980) 419-432.

[21] S. Vaddiraju, H. Chandrasekaran, M.K. Sunkara, Vapor Phase Synthesis of Tungsten Nanowires, J. Am. Chem. Soc. 125 (2003) 10792-10793. http://dx.doi.org/10.1021/ja035868e.

[22] J.-Y. Kim, D. Jang, J.R. Greer, Tensile and compressive behavior of tungsten, molybdenum, tantalum and niobium at the nanoscale, Acta Mater. 58 (2010) 2355-2363. https://doi.org/10.1016/j.actamat.2009.12.022.

[23] J.R. Greer, D. Jang, X.W. Gu, Exploring Deformation Mechanisms in Nanostructured Materials, JOM 64 (2012) 1241-1252. https://doi.org/10.1007/s11837-012-0438-6.

[24] I.V. Shvets, R. Wiesendanger, D. Bürgler, G. Tarrach, H.-J. Güntherodt, J.M.D. Coey, Progress towards spinpolarized scanning tunneling microscopy, J. Appl. Phys 71 (1992) 5489-5499. http://dx.doi.org/10.1063/1.350522. 
[25] S.F. Ceballos, G. Mariotto, S. Murphy, I.V. Shvets, Fabrication of magnetic STM probes and their application to studies of the $\mathrm{Fe}_{3} \mathrm{O}_{4}(001)$ surface, Surf. Sci. 523 (2003) 131-140. https://doi.org/10.1016/S0039-6028(02)024585.

[26] N. Berdunov, S. Murphy, G. Mariotto, I.V. Shvets, Atomically Resolved Spin-Dependent Tunneling on the $\begin{array}{lllllll}\text { Oxygen-Terminated } & \mathrm{Fe}_{3} \mathrm{O}_{4}(111), \quad \text { Phys. } & \text { Rev. } & \text { Lett. } & 93 & \text { (2004) } & 057201 .\end{array}$ https://doi.org/10.1103/PhysRevLett.93.057201.

[27] S.I. Bozhko, S.V. Chekmazov, V. Usov, O. Lübben, A.M. Ionov, H.-C. Wu, V.N. Semenov, M.E. Nesterova, S.A. Krasnikov, I.V. Shvets, Composite $\mathrm{Fe}_{3} \mathrm{O}_{4}-\mathrm{W}(100)$ probes for scanning tunneling microscopy, J. Appl. Phys. 122 (2017) 235301. https://doi.org/10.1063/1.5001057.

[28] S. Lee, A. Fursina, J.T. Mayo, C.T. Yavuz, V.L. Colvin, R.G.S. Sofin, I.V. Shvets, D. Natelson, Electrically driven phase transition in magnetite nanostructures, Nat. Mater. 7 (2008) 130-133. https://doi.org/10.1038/nmat2084.

[29] V.G. Glebovsky, V.N. Semenov, V.V. Lomeyko, Influence of the crystallization conditions on the structural perfection of molybdenum and tungsten single crystals, J. Cryst. Growth 87 (1988) 142-150. https://doi.org/10.1016/0022-0248(88)90353-3.

[30] S.I. Bozhko, V.G. Glebovsky, V.N. Semenov, I. Smirnova, On the growth of tungsten single crystals of high structural quality, J. Cryst. Growth 311 (2008) 1-6. https://doi.org/10.1016/j.jcrysgro.2008.09.175.

[31] E. Lassner, W.-D. Schubert, Tungsten. Properties, Chemistry, Technology of the Element, Allows, and Chemical Compounds, Kluwer Academic / Plenum Publishers, New York, 1999.

[32] Yu.V. Baranov, The Joffe effect on metals, Rus. Moscow State Industrial University (MSIU), Moscow, 2005.

[33] Yu.V. Baranov, Study of the surface role in the formation of physical and mechanical properties of the single crystalline tungsten during deformation, Rus. Deformatsiya i Razrushenie materialov 7 (2007) 40-42.

[34] M. Šob, L.G. Wang, V. Vitek, Theoretical tensile stress in tungsten single crystals by full-potential firstprinciples calculations, Mat. Sci. Eng. A - Struct. 234 - 236 (1997) 1075-1078. https://doi.org/10.1016/S09215093(97)00329-8.

[35] D. Roundy, C.R. Krenn, M.L. Cohen, J.W. Morris, Jr., The ideal strength of tungsten, Philos. Mag. A 81 (2001) 1725-1747. https://doi.org/10.1080/01418610108216634.

[36] Y.-L. Liu, H.-B. Zhou, Y. Zhang, S. Jin, G.-H. Lu, The ideal tensile strength and deformation behavior of tungsten single crystal, Nucl. Instrum. Meth. B 267 (2009) 3282-3285. https://doi.org/10.1016/j.nimb.2009.06.088.

[37] S. Giusepponi, M. Celino, The ideal tensile strength of tungsten and tungsten alloys by first-principles calculations, J. Nucl. Mater. 435 (2013) 52-55. https://doi.org/10.1016/j.jnucmat.2012.12.028. 
[38] K.A. Bukreeva, A.M. Iskandarov, S.V. Dmitriev, Y. Umeno, R.R. Mulyukov, Theoretical Shear Strength of FCC and HCP Metals, Phys. Solid State 56 (2014) 423-428. https://doi.org/10.1134/S1063783414030081.

[39] A. Joffé, M.W. Kirpitschewa, M.A. Lewitzky, Deformation und Festigkeit der Kristalle, Z. Phys. 22 (1924) 286-302. https://doi.org/10.1007/BF01328133.

[40] A. Joffé, M. Lewitzky, Über die Festigkeit und Elastizitätsgrenze des natürlichen Steinsalzes, Z. Phys. 31 (1925) 576-583. https://doi.org/10.1007/BF02980612.

[41] A.F. Joffe, Physics of crystals, Sov. Leningrad State Publishing House, Leningrad, 1929.

[42] A.F. Joffe, M.V. Kirpitcheva, M.A. Levitskaya, Deformation and strength of crystals, Sov. Phys. Uspekhi 93 (1967) 303-314. https://doi.org/10.3367/UFNr.0093.196710e.0303.

[43] A.A. Griffith, VI. The Phenomena of Rupture and Flow in Solids, Philos. Tr. Soc. Lond. 221 (1921) $163-198$. https://doi.org/10.1098/rsta.1921.0006.

[44] F. Zwicky, Die Reißfestigkeit von Steinsalz, Phys.Z. 24 (1923) 131-137.

[45] A.B. Lebedev, S.A. Pul'nev, V.V. Vetrov, Yu.A. Burenkov, V.I. Kopylov, K.V. Betekhtin, Thermal stability of submicrocrystalline copper strengthened with $\mathrm{HfO}_{2}$ nanoparticles in the temperature range $20-500{ }^{\circ} \mathrm{C}$, Phys. Solid. State 40 (1998) 1155-1157. https://doi.org/10.1134/1.1130509.

[46] Y. Kraftmakher, Lecture notes on EQUILIBRIUM POINT DEFECTS and THERMOPHYSICAL PROPERTIES OF METALS, World Scientific Publishing Co. Pte. Ltd., Singapore, 2000.

[47] V.Ya. Chekhovskoi, V.E. Peletsky, On Relaxation of Point Defects in Metals, High Temp. 49 (2011) $45-54$. https://doi.org/10.1134/S0018151X10051013.

[48] F.R.N. Nabarro, Deformation of Crystals by the Motion of Single Ions, in: Report of a Conference on Strength of Solids, The Physical Society, London, 1948, pp. 75-90.

[49] C. Herring, Diffusional Viscosity of a Polycrystalline Solid, J. Appl. Phys. 21 (1950) 437-445. https://doi.org/10.1063/1.1699681.

[50] Z. Zhang, S. Satpathy, Electron states, magnetism, and the Verwey transition in magnetite, Phys. Rev. B 44 13319-13331 (1991). https://doi.org/10.1103/PhysRevB.44.13319.

[51] J.G. Tobin, S.A. Morton, S.W. Yu, G.D. Waddill, I.K. Schuller, S.A. Chambers, Spin resolved photoelectron spectroscopy of $\mathrm{Fe}_{3} \mathrm{O}_{4}$ : the case against half-metallicity, J. Phys. - Condens. Mat. 19 (2007) 315218. https://doi.org/10.1088/0953-8984/19/31/315218.

[52] K. Radikan, N. Berdunov, G. Manai, I.V. Shvets, Epitaxial molybdenum oxide grown on Mo(110): LEED, STM, and density functional theory calculations, Phys. Rev. B $75 \quad(2007) \quad 155434$. https://doi.org/10.1103/PhysRevB.75.155434. 
[53] O. Lübben, S.A. Krasnikov, A.B. Preobrajenski, B.E. Murphy, S.I. Bozhko, S.K. Arora, I.V. Shvets, Selfassembly of $\mathrm{Fe}$ nanocluster arrays on templated surfaces, J. Appl. Phys. 111 (2012) $07 \mathrm{~B} 515$. https://doi.org/10.1063/1.3676207. 